\title{
Efficient Synthesis of Polyacrylamide based Hydrogels using Serrated Open Blade Static Mixers
}

\author{
Chandra Mouli R. Madhuranthakam ${ }^{1}$, Sudhanshu S. Soman ${ }^{2}$ \\ ${ }^{1}$ Chemical Engineering Department, Abu Dhabi University \\ P.O.Box 59911, Abu Dhabi, UAE \\ chandra.mouli@adu.ac.ae; s4soman@uwaterloo.ca \\ ${ }^{2}$ Chemical Engineering Department, University of Waterloo \\ Waterloo, Ontario, Canada, N2L 3G1
}

\begin{abstract}
Polyacrylamide based hydrogels are widely used in enhanced oil recovery applications where huge amounts of polymer is injected in the tertiary stage after flooding with water and steam. Synthesis of these polymers heavily depends on the mixing efficiency of the agitators in addition to the reactor design itself. The high viscosity of the medium even at a very low polymer loading makes it inevitable to operate the reactors/reaction systems using diluted polymer solution. To overcome this problem, we proposed to use a static mixer with open blade structure such as Sulzer SMX for continuous production of these water soluble polymers. Further, to achieve enhanced mixing efficiency, the internal structure of this static mixer is modified by introducing perforations and serrations on and along the blades of the internal element. Three different type of serrations such as triangular, square and semi-circular geometries were investigated. The semi-circular serration showed better performance over others and is used for conducting the free radical polymerization of acrylamide. The geometry of the internal structure is designed using AutoCAD which is imported to COMSOL environment where the reaction domain is meshed and solved using finite element method. It was observed that enhanced mixing and conversion of acrylamide were achieved. The success of using the proposed modified static mixer can also be extended and easily used for synthesis of hydrolyzed, partially hydrolyzed and copolymers of acrylamide.
\end{abstract}

Keywords: Polyacrylamide, Hydrogel, Geometry Effects, Static Mixer, CFD Simulation.

\section{Introduction}

Static mixers have a wide range of industrial applications and are predominantly used in polymer industry including blending of polymers, synthesis of in-situ nanocomposites and nanoparticles, polymer extrusion process, emulsification processes and in the production of many commodity polymers[1,2].

The choice of the reactor in the synthesis of polymer depends on the reaction kinetics and viscosity of the polymer. Continuous stirred tank reactor (CSTR) is generally used for the homogenous polymerization reaction with high termination rate, since it gives narrowest molecular weight distribution and higher conversion. On the other hand, tubular reactor can be used for the continuous bulk polymerization of commodity polymers due to its simplicity and low cost of operation as there are no moving parts. However due to high viscosity of reaction mixture, poor radial mixing is achieved which is a major drawback of using a tubular reactor for polymerization. This in turn results into higher conversion of monomer near the walls where velocities are very low and lower conversions at the center where velocity is maximum. This problem can be overcome by using static mixers in polymerization process. Since static mixers provide higher axial and radial mixing, almost plug flow behavior is achieved resulting into narrower molecular weight distribution of polymer. An additional benefit would be, continuous production of polymers can be achieved by incorporating static mixers.

Use of static mixers in the industrial production of polymers has increased tremendously. Amidst all, the synthesis of polyacrylamide has gained significant interest due to its application in enhanced oil recovery (EOR). In this process, static mixers are used to mix partially hydrolyzed polyacrylamide (HPAM) with injected water to recover the oil from reservoir. Since this polymer solution is extremely viscous, static mixers are used to perform this operation. SMs are commercially proven best choice to carry out this type of operations in EOR process. Though polyacrylamide is used to a large extent in EOR, maximum consumption of polyacrylamide is in the process of flocculating solids in a liquid. Largest use of polyacrylamide is in water treatment, paper making and screen printing. With the improving technology, synthesis of PAM 
process has started incorporating the use of static mixers in production process. However, it is restricted to lab scale only. This research article facilitates a working CFD model for the synthesis of polyacrylamide using static mixers. Given the fact that actual homopolymerization of acrylamide involves several reactions, this CFD model has included major reactions only such as initiation, propagation and termination. Current CFD model has included as many features as possible to mimic the real experimental process. In the current work, synthesis of polyacrylamide was performed using the SMX static mixer. Further, by modifying the internal geometry of the SMX static mixer its mixing performance can be enhanced and was shown through CFD simulations by Soman et al.[3]. In this work, the performance with respect to polymerizing acrylamide using a circular serrated SMX geometry is realized through simulations using COMSOL software. The circular modifications are proven to be the best among all the modified static mixer geometries considered for this study [3]. The performance of both static mixers has been analyzed based on the final polymer concentration, reaction rate and monomer conversion. Comparison of results is shown in terms of contour plots and line plots. In the next part, brief description about the materials and method of polymerization used for CFD model for acrylamide polymerization is discussed.

\section{CFD Model for Homopolymerization of Acrylamide}

To characterize the flow field behavior in all modified SM geometries, all the numerical simulations are performed using Computational Fluid Dynamics (CFD) software COMSOL Multiphysics 5.0. All modified SM geometries are created using AutoCAD. These geometries are then imported to COMSOL environment and discretized into small control volumes using tetrahedral mesh. Acrylamide monomer undergoes polymerization by redox initiation. Polymerization can also be induced by photo initiation, radiation, electron initiation and by ultrasonic waves [4]. However, the principal method of industrial production of PAM and its derivatives is either free-radical homopolymerization in solution or emulsion polymerization [4, 5]. This model gives an insight of actual homopolymerization experiment in case of two different static mixer geometries. In this study, polymerization of acrylamide is initiated by activating the monomer by Potassium Persulfate initiator. The concentration of initiator was $5.21 \times 10^{-2}$ moles/liter, while the monomer concentration was $0.04 \mathrm{~mol} / \mathrm{L}$. For all the simulations, isothermal conditions have been adopted and polymerization temperature is assumed to be constant at $30^{\circ} \mathrm{C}$.

\subsection{Reaction and Kinetics of Polymerization}

For the homopolymerization of acrylamide, the reaction kinetics can be written in terms of individual steps of reaction mechanism. Consumption of initiator and thereby the continuous decrease in initiator concentration can be represented according to the first order expression as shown by eq. (1).

$$
\left[\mathrm{I}_{2}\right]=\left[\mathrm{I}_{2}\right]_{0} \exp \left(-\mathrm{k}_{\mathrm{d}} \mathrm{t}\right)
$$

where $\left[\mathrm{I}_{2}\right]$ is the initiator concentration, $\mathrm{k}_{\mathrm{d}}$ is the disassociation rate constant and $\mathrm{t}$ is the time. Since the decomposition of initiator and the radical generation occurs in two steps according to eq.(1) and (2) respectively, the rate equation for the chain propagation can be written in two different forms. When eq. (1) is assumed to be rate limiting for the polymerization, the rate expression can be written as

$$
\mathrm{dRdt}=2 \mathrm{FkdI} 2-\mathrm{ktR} 2
$$

Where, $\mathrm{R}$ is the radical concentration, $\mathrm{k}_{\mathrm{t}}$ is the termination rate constant, $\mathrm{F}$ is the fraction of radicals which initiates the chain propagation. When eq. (2) is considered to be rate limiting, the rate equation for propagating radical [R] is written as

$$
\frac{\mathrm{d}[\mathrm{R}]}{\mathrm{dt}}=\mathrm{k}_{\mathrm{i}}\left[\mathrm{I}^{*}\right][\mathrm{m}]-\mathrm{k}_{\mathrm{t}}[\mathrm{R}]^{2}
$$

where $\mathrm{I}^{*}$ is the disassociated initiator, $\mathrm{m}$ is the monomer and $\mathrm{k}_{\mathrm{i}}$ is the initiator rate constant The rate equation for the monomer concentration is represented as 


$$
\frac{\mathrm{d}[\mathrm{m}]}{\mathrm{dt}}=-2 \mathrm{Fk}_{\mathrm{d}}\left[\mathrm{I}_{2}\right]-\mathrm{k}_{\mathrm{p}}[\mathrm{R}][\mathrm{m}]
$$

where $\mathrm{k}_{\mathrm{p}}$ is the propagation rate constant It is necessary to consider only those initiator radicals which add the monomer and activates the chain propagation. Some initiating radicals recombine with other radicals or they decompose into non-initiating radicals. For this reason, only very small fraction ' $F$ ' of initiator concentration contributes to chain polymerization and thereby, the rate equation of monomer concentration can be written as

$$
\frac{\mathrm{d}[\mathrm{m}]}{\mathrm{dt}}=-\mathrm{k}_{\mathrm{p}} \sqrt{\frac{2 \mathrm{Fk}_{\mathrm{d}}\left[\mathrm{I}_{2}\right]}{\mathrm{k}_{\mathrm{t}}}}[\mathrm{m}]
$$

The eq. (5) shows the overall rate of free radical polymerization is proportional to monomer concentration and to the square root of initiator concentration. Once the reaction rate expression is determined in term of monomer and initiator concentration, values of reaction rate parameters are included in the CFD model. For the reaction rate shown in eq. (5), the value of overall reaction rate constant $\mathrm{k}$ at $30^{\circ} \mathrm{C}$ is

$$
\mathrm{k}=\mathrm{k}_{\mathrm{p}} \sqrt{\frac{2 \mathrm{Fk}_{\mathrm{d}}}{\mathrm{k}_{\mathrm{t}}}}=0.108 \pm 0.003 \frac{\text { liter }^{0.75}}{\text { mole }^{0.75} \mathrm{~min}}
$$

In the CFD model, the kinetics parameters can also be expressed as Arrhenius expression, which is

$$
\mathrm{k}=1.70 \times 10^{11} \exp \left(\frac{-16900}{\mathrm{RT}}\right) \frac{\operatorname{liter}^{0.75}}{\mathrm{~mole}^{0.75} \min }
$$

In the current CFD model of polymerization of acrylamide, polymerization reactions taking place inside the SMX and circular serrated SMX geometries are coupled with the reaction kinetics and flow conditions of reactants and resulting polymer. For all the simulations, laminar flow conditions of monomer and initiator fluid streams are used. As polyacrylamide is non-Newtonian fluid, the viscosity of polymer increases with the increasing molecular weight. The viscosity expression for the generation of polyacrylamide solution is given by Huggins eq. (8).

$$
\frac{\mu_{\mathrm{sp}}}{\mathrm{c}}=(\mu)+\mathrm{k}^{\prime}(\mu)^{2} \mathrm{c}
$$

Where $\mu_{\mathrm{sp}}$ is the pure solvent viscosity, $\mathrm{k}^{\prime}$ is the Huggins constant and $\mathrm{c}$ is the solution concentration. In the next section, the results of numerical simulations for the polyacrylamide are shown in the form of polymer concentration, reaction rate monomer conversion.

\section{Results}

The CFD model for homopolymerization of acrylamide has been solved for two cases: 1) SMX static mixer and 2) Circular serrated SMX geometry. Since the circular serrated SMX geometry gives the best performance among all modified static mixer geometries, it has been chosen for homopolymerization of acrylamide. Though same dimensions of the static mixer geometries are used for the polymerization, it must be mentioned that additional inlet for the injection of initiator has been provided in both the cases. In Fig. 1 and Fig. 2, static mixer geometries used in the simulations are shown. The purpose of providing additional inlet port (inlet 2) is to inject the initiator stream inside the reactor, whereas monomer is injected from the main pipe inlet only. 


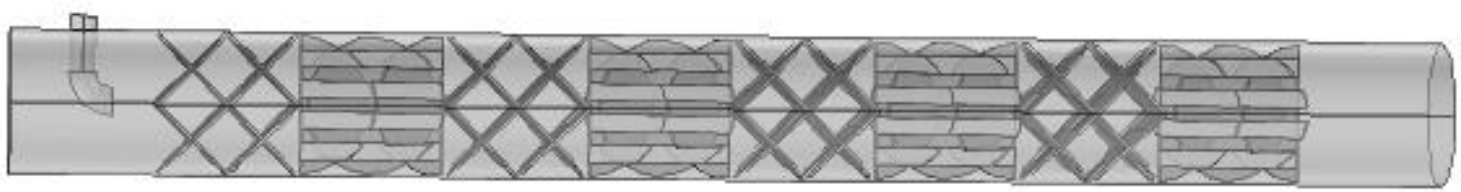

Fig. 1: SMX static mixer geometry 8 elements with additional pipe inlet for the initiator inflow.

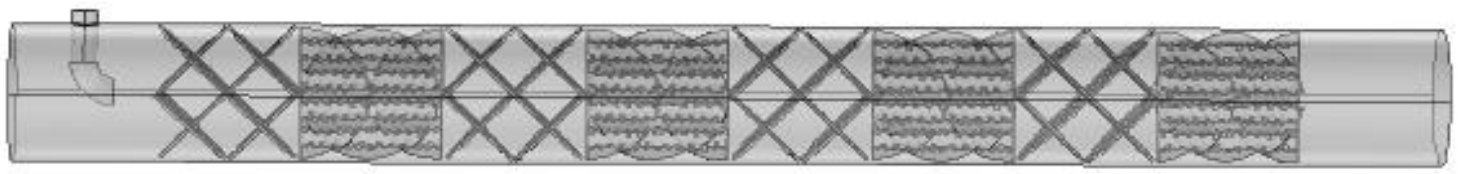

Fig. 2: Circular serrated SMX static mixer geometry 8 elements with additional pipe inlet for the initiator flow.

The results for the chemical reaction along with the flow field calculations are shown from Fig. 3 to Fig. 5 for SMX and Circular serrations. These figures show the streamlines of the flow field for a Reynolds number of 30 for both geometries. The colour expression represents the concentration change in the polymer product. Colour scale from blue to red represents the minimum to maximum concentration of the resulting polymer.

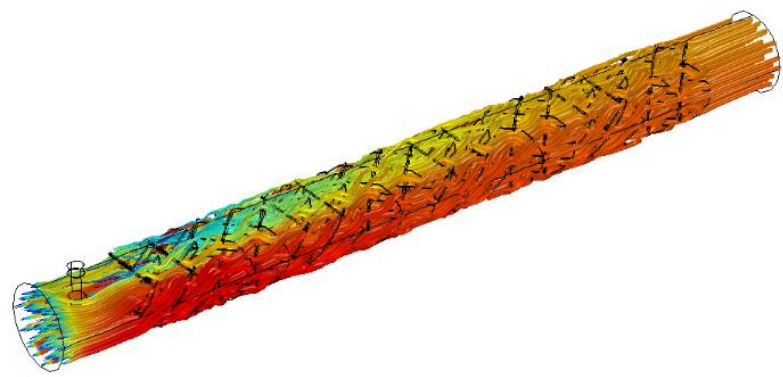

(a)

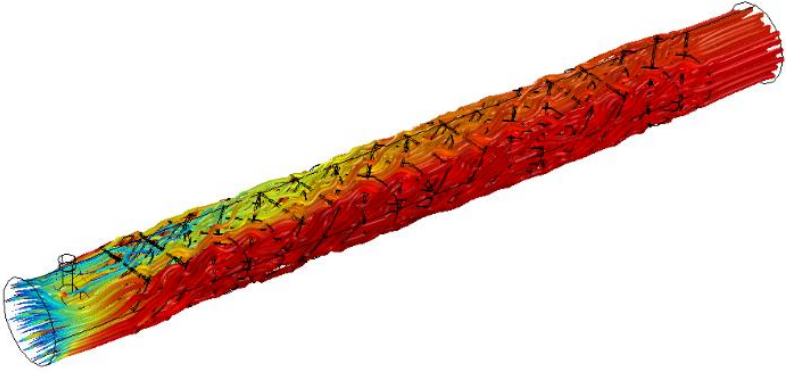

(b)

Fig. 3: Comparison of concentration of resulting polyacrylamide for (a) SMX geometry (b) Circular serrated SMX geometry.

From the streamlines plots shown in Fig. 3 to Fig. 5, the mixing effects are evident in the region where second inlet port for initiator is provided. In both the cases, the polymerization reaction starts as soon as the initiator comes into contact with the monomer. From Fig. 3, it is apparent that due to the higher mixing ability of circular serrations, the polymerization reaction starts early and attains higher polymer concentration values than the SMX geometry. Further, Fig. 4 and Fig. 5 shows the streamline plots for both the geometries in the form of initiator concentration and monomer concentration respectively. 


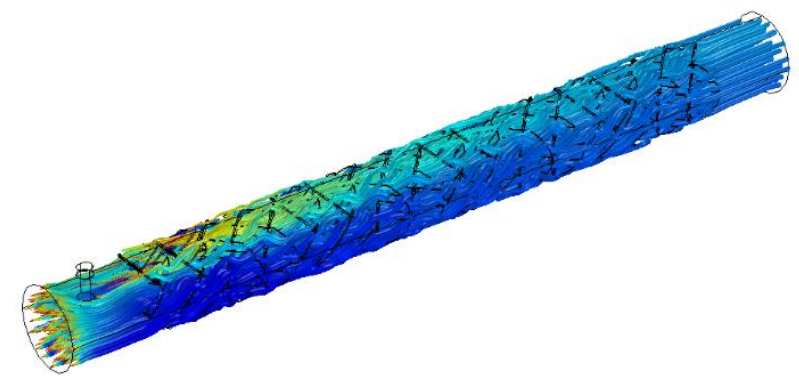

(a)

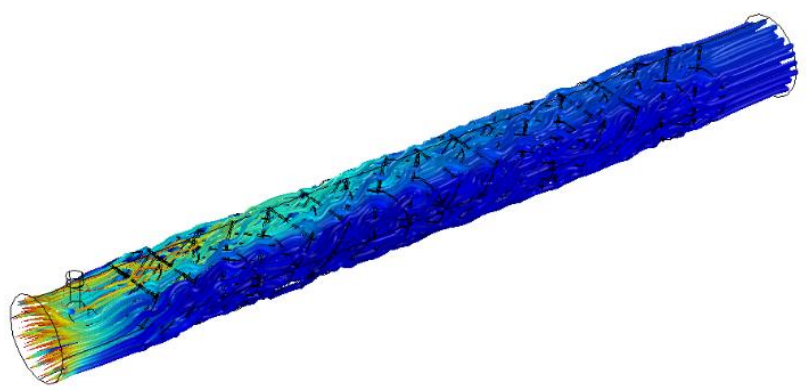

(b)

Fig. 4: Comparison of concentration of initiator for (a) SMX geometry (b) Circular serrated SMX geometry.

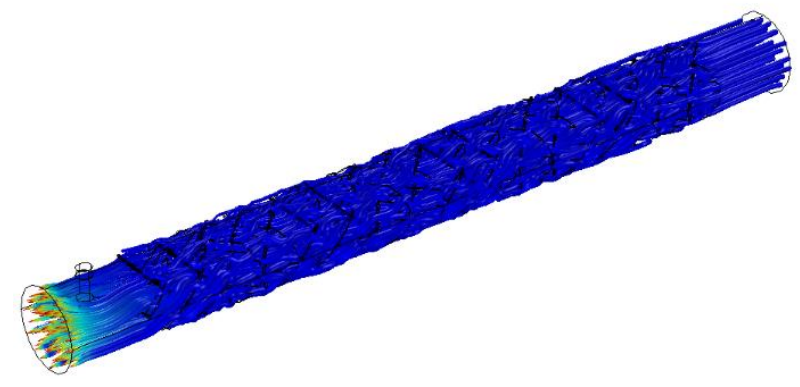

(a)

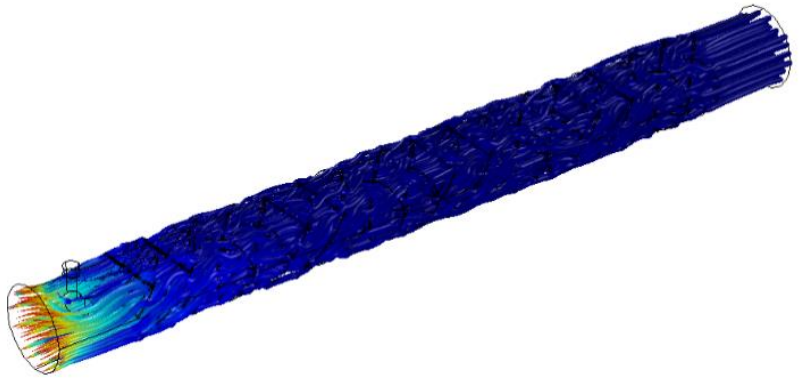

(b)

Fig. 5: Comparison of concentration of monomer for (a) SMX geometry (b) Circular serrated SMX geometry.

During the polymerization, the reactant monomer and initiator are consumed along the length of the reactor. The injection stream of second inlet port of initiator mixes with the main monomer stream and results into non-uniform distribution of initiator in the SMX geometry (shown as the blue stream in Fig. 4 (a), which is longer). Depending on the degree of mixing of initiator and monomer, the rate of generation of polymer varies. Fig. 5 (a) shows that SMX geometry has relatively higher monomer concentration in the flow domain, which implies relatively less monomer is converted during the polymerization. Mixing is more effective in the circular serrations, therefore initiator distribution is relatively more uniform (shown as the blue stream of initiator in Fig. 4 (b) which is shorter). In case of circular serrated SMX geometry relatively lower monomer concentration is observed, which indicates more consumption of monomer in producing higher concentration of polymer. The purpose of comparing the streamline plots was to demonstrate the reaction kinetics coupled with the flow field of injected reactant streams. However, they are not sufficient to show that the circular serrated SMX improves the homopolymerization of acrylamide. In order to analyze the actual static mixer performance for polymerization system, comparison is shown in terms of reaction rate and monomer conversion for both the reactors. Fig. 6 and Fig. 7 shows line plots for the variation of reaction rate along the axial length of the reactor for SMX and circular serrations respectively. 


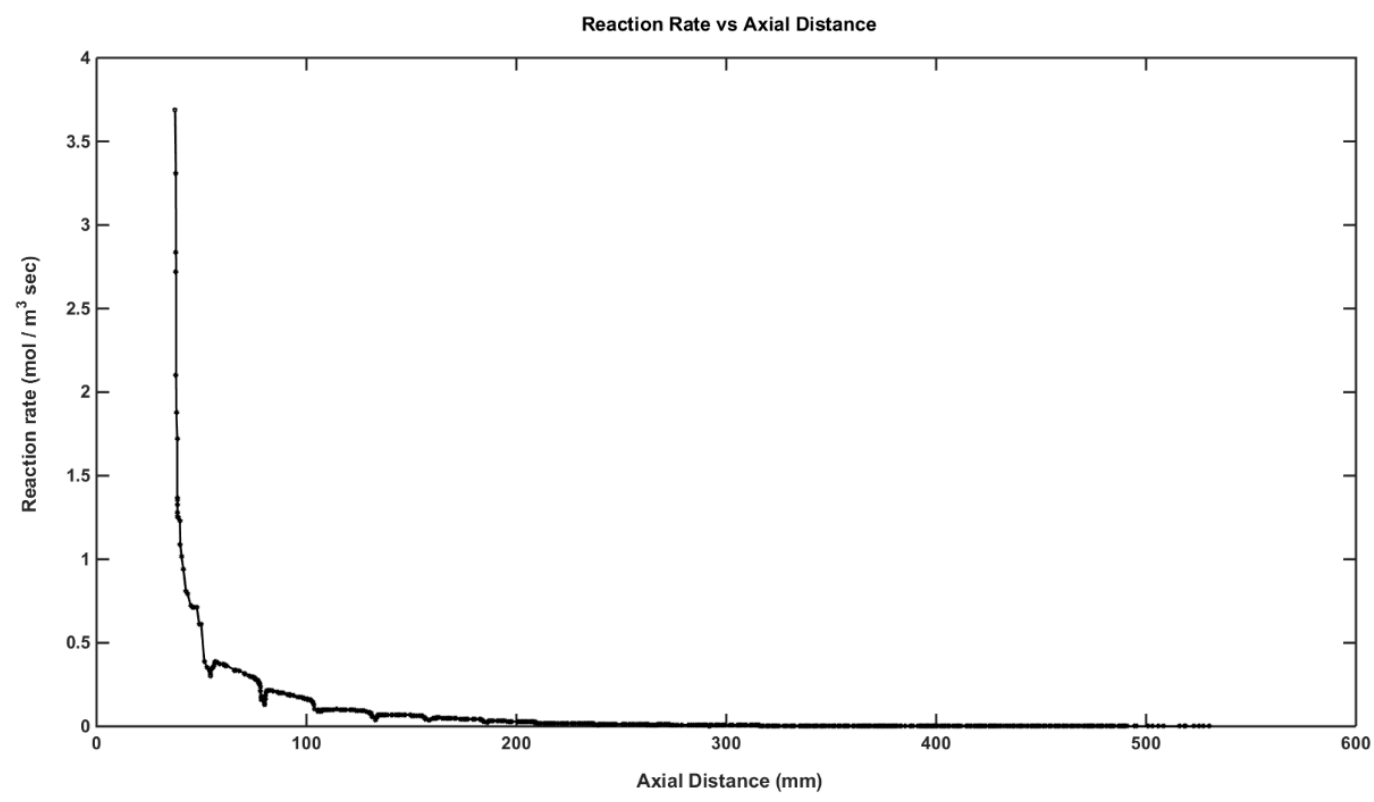

Fig. 6: Variation in Reaction rate along the length of reactor for SMX geometry.

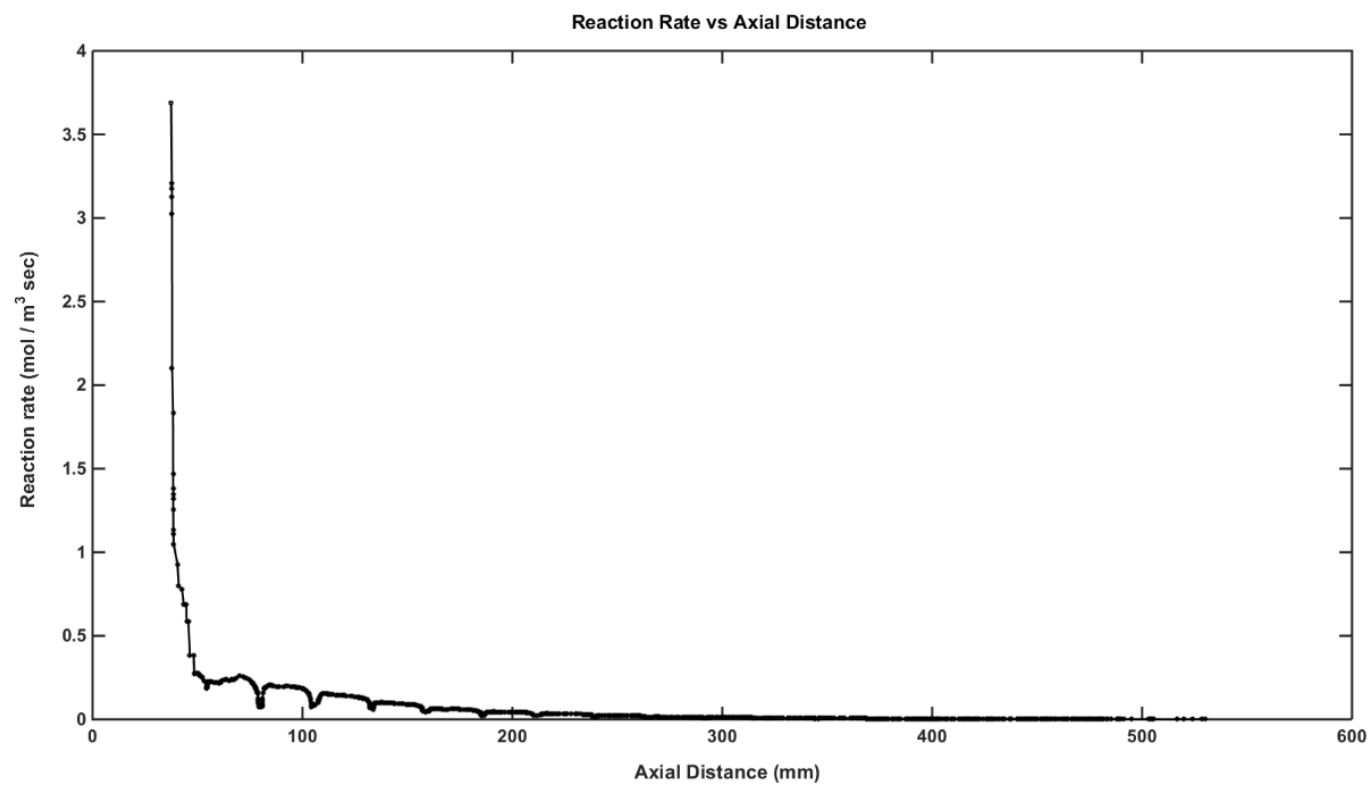

Fig. 7: Variation in Reaction rate along the length of reactor for Circular serrated SMX geometry.

Rapid decrease in the reaction rate values shows that monomer and initiator are consumed immediately near the inlet section and generates higher concentrations of polyacrylamide. These results are in good agreement with the streamline plots shown in Fig. 3 - Fig. 5 , where higher concentration gradients of monomer and initiator are present in the inlet region and indicates higher consumption of reactants. It should be noted that since initiator is injected from the second inlet, there is no reaction occurring in that region. Due to this, polymer generation starts after the second inlet port when initiator starts mixing with the monomer.

Rapid changes in reaction rate plots indicates the movement of fluid streams through the static mixers. As the fluid stream of initiator and monomer moves forward through the mixer elements, it gets split, rotated and mixed. This type of 
fluid stream movement results into sudden reaction rate gradients. Fig. 7 shows higher reaction rate gradients as compared to that of shown in Fig. 6, which also implies better mixing of monomer and initiator in circular serrated SMX than standard SMX geometry. Higher the mixing, higher is the contact between monomer and initiator which gives more polymer yield. Concentration of monomer and initiator decreases along the length of the reactor, which reduces the rate of polymerization. Zero reaction rate indicates that monomer is completely converted into polymer. Therefore it is desired to achieve reaction rate values approaching zero. All the plots of reaction rates are directly correlated to the plots of monomer conversion, which is shown by eq. (9).

$$
\text { Conversion }=1-\left(\frac{\mathrm{M}}{\mathrm{M}_{0}}\right)
$$

Where $\mathrm{M}$ is the monomer conversion at any point in the reactor and $\mathrm{M}_{0}$ is the initial monomer concentration. Fig. 8 and Fig. 9 shows the plots of monomer conversion for SMX and circular SMX geometry.

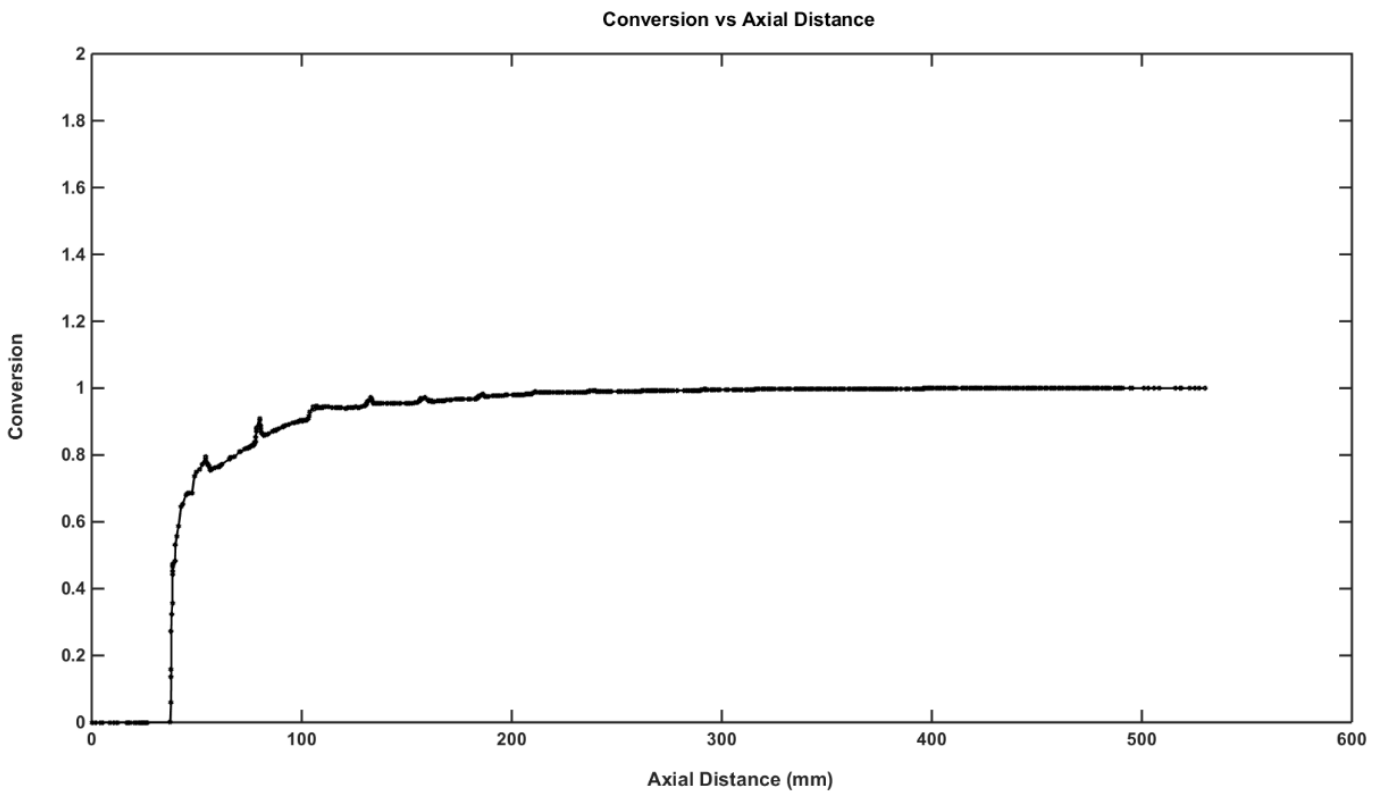

Fig. 8: Variation in monomer conversion along the length of reactor for SMX geometry. 


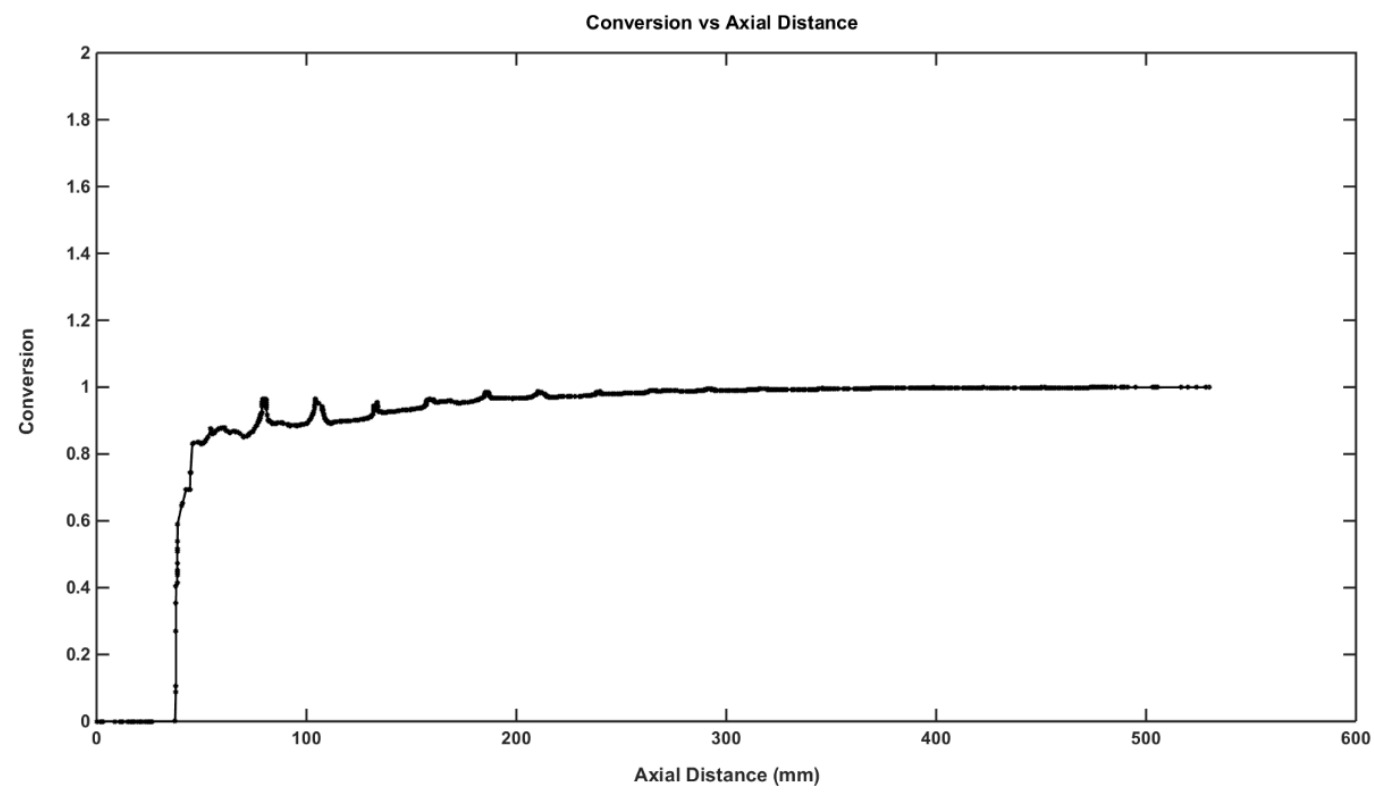

Fig. 9: Variation in monomer conversion along the length of reactor for Circular serrated SMX geometry.

Similar to the reaction rate plots, the plots shown in Fig. 8 and Fig. 9 have rapid change in quantities near the inlet region and typical oscillations past the secondary inlet port. From the comparison of Fig. 8 and Fig. 9, it is clear that monomer and initiator fluid streams are mixed very well in case circular serrations and hence results into higher monomer conversion values than the SMX geometry. Furthermore, to compare the performance of both the geometries, volume average polymer concentration, reaction rate and monomer conversion values have been listed in the Table 1.

Table 1: Comparison of parameters to analyze the performance of SMX and Circular serrated SMX.

\begin{tabular}{|l|l|l|}
\hline & SMX & Circular serrated SMX \\
\hline Polymer concentration $\left(\mathrm{mol} / \mathrm{m}^{3}\right)$ & 31.426 & 33.102 \\
\hline Reaction rate $\left(\mathrm{mol} / \mathrm{m}^{3} \mathrm{sec}\right)$ & 0.13976 & 0.048119 \\
\hline Conversion & $93.353 \%$ & $97.701 \%$ \\
\hline
\end{tabular}

\section{Conclusion}

The main intention of designing this CFD model was to show the benefits of using modified static mixers for polymerization of acrylamide based monomers. The homopolymerization of acrylamide was virtually simulated by mimicking the experimental conditions as closely as possible. By comparison of certain parameters like monomer conversion, reaction rate and volume average polymer concentrations, it can be concluded that circular serrated SMX geometry gives better performance than the SMX static mixer. Higher monomer conversion and hence higher polymer concentration was achieved in the case of circular serrated SMX mixer. Final comparison shown in Table 1 clearly indicates higher polymer yield can be achieved using circular SMX geometry for homopolymerization of acrylamide.

\section{References}

[1] L. Zhang, J. Dong, B. Jiang, Y. Sun, F. Zhang, L. Hao, “ A study of mixing performance of polyacrylamide solutions in a new-type static mixer combination," Chem. Eng. Process. Process Intensif., vol. 88, pp. 19-28, 2015.

[2] C. M. R. Madhuranthakam, Q. Pan, G. L. Rempel, "Residence time distribution and liquid holdup in Kenics KMX static mixer with hydrogenated nitrile butadiene rubber solution and hydrogen gas system," Chem. Eng. Sci., vol. 64, pp. 3320-3328, 2009. 
[3] S. Soman and C. M. R. Madhuranthakam, "Effects of internal geometry modifications on the dispersive and distributive mixing in static mixers," Chem. Eng. Process. Process Intensif, vol. 122, pp. 31-43, 2017.

[4] V. F. Kurenkov and L. I. Abramova, "Homogeneous Polymerization of Acrylamide in Solutions," Polym. Plast. Technol. Eng., vol. 31, no. 7-8, pp. 659-704, 1992.

[5] S. R. Sandler and W. Karo, "Polymer Synthesis," Polym. Synth., pp. 354-414, $1996 .$.

[6] W. F. R. Ahmet Giz, Huceste Çatalgil-Giz, Alina Alb, Jean-Luc Brousseau, "Kinetics and mechanisms of acrylamide polymerization from absolute, online monitoring of polymerization reaction," Macromolecules, vol. 34, no. 5, pp. 1180-1191, 2001. 